\title{
Inverse of Interval Matrix and Solution of System of Equations with Interval Coefficient (Using Modified Interval Arithmetic)
}

\author{
Reema Gupta \\ Department of Mathematics, IIT Kharagpur
}

In this paper, a method to compute the inverse of an interval matrix is studied based on the modified interval arithmetic and solving system of equations with interval coefficients using Determinant Method, Jacobi Iterative Method, Crout Method, Cholesky Method. And also developed a Matlab code for Inverse of an Interval Matrix. If an interval linear system of equations is solved by existing interval arithmetic method the replacing solution in interval system of equations, the interval width is more than the interval width of right hand side intervals. On the other hand, applying modified interval arithmetic the interval width is less than interval width than previously obtained by existing interval arithmetic.

Keywords :

Existing interval arithmetic, Modified interval arithmetic, Interval matrix, Inverse matrix.

\section{Introduction}

It is well known, that matrices play a major role in various areas such as science, engineering and technology, social sciences and many others. In real life, due to the inevitable measurement inaccuracy, we do not know the exact values of the measured quantities; we know, at best, the intervals of possible values. Consequently, we can not successfully use traditional classical matrices and hence the use of interval matrices is more appropriate.

Following notations are being used through out the paper:

- $\mathbb{I} R=\left\{A=\left[\mathrm{a}_{1}, a_{2}\right]: a_{1} \leq a_{2}\right.$ and $\left.a_{1}, a_{2} \in \mathbb{R}\right\}$ be the set of all proper intervals,

- $\overline{\mathbb{I R}}=\left\{\mathrm{A}=\left[a_{1}, a_{2}\right]: a_{1}>a_{2}\right.$ and $\left.a_{1}, a_{2} \in \mathbb{R}\right\}$ be the set of all improper intervals on the real line $\mathbb{R}$,

- mid-point $m(A)=\left(a_{1}+a_{2}\right) / 2$

- width(or half-width) $w(A)=\left(a_{2}-a_{1}\right) / 2$

- The set of generalized intervals(proper and improper) by $\mathbb{D}$

$$
\mathbb{D}=\mathbb{I} \mathbb{R} \cup \overline{\mathbb{I}}=\left\{\left[a_{1}, a_{2}\right]: a_{1}, a_{2} \in \mathbb{R}\right\}
$$

- $\operatorname{dual}(\mathrm{A})=\operatorname{dual}\left[a_{1}, a_{2}\right]=\left[a_{2}, a_{1}\right]$

- $\operatorname{opp}\left\{\left[a_{1}, a_{2}\right]\right\}=\left[-a_{1},-a_{2}\right]$
Interval arithmetic is used with error analysis.

Rounding error analysis : Consider the expression $f(x)=1-x+x^{2}$ with $x=0.531$, i.e with $10^{-3}$ precision. Computing this expression with classical arithmetic gives the result $f(x)=0.610$. Now, if we perform the computations using Interval arithmetic, we get $f(x)=0.469+\frac{(0.531)^{2}}{2} \in 0.469+$ $\frac{[0.281,0.282]^{2}}{2}$ and so $f(x) \in 0.469+[0.140,0.141]=$ $[0.609,0.610]$. This guarantees that the exact result is within the interval $[0.609,0.610]$.

\subsection{Existing Interval Arithmetic}

For $X=\left[x_{1}, x_{2}\right], Y=\left[y_{1}, y_{2}\right]$

- $X \odot Y=\{x \odot y: x \in X, y \in Y\}$, for $\odot \in$ $\{+,-, \cdot, /\}$

- $X+Y=\left[x_{1}+y_{1}, x_{2}+y_{2}\right]$

- $X-Y=\left[x_{1}-y_{2}, x_{2}-y_{1}\right]$

- $X \cdot Y=[\min T, \max T]$, where $T=\left(x_{1} y_{1}, x_{1} y_{2}\right.$, $\left.x_{2} y_{1}, x_{2} y_{2}\right)$

- $\frac{X}{Y}=X \cdot\left(\frac{1}{Y}\right)$, where $\frac{1}{Y}=\left\{y: \frac{1}{y} \in Y\right\}=$ $\left[\frac{1}{y_{2}}, \frac{1}{y_{1}}\right]$

\subsection{Modified Interval Arithmetic}

For $X=\left[x_{1}, x_{2}\right], Y=\left[y_{1}, y_{2}\right] \in \mathbb{D}$ and for $* \in\{+,-, \cdot, /\}$

we define $X * Y=[m(X) * m(Y)-k, m(X) * m(Y)+k]$ where $k=\min \{(m(X) * m(Y))-\alpha, \beta-(m(X) * m(Y))\}$ $\alpha$ and $\beta$ are the end points of the interval $X * Y$ 
under the existing interval arithmetic. In particular

\section{(i) Addition :}

$X+Y=\left[x_{1}, x_{2}\right]+\left[y_{1}, y_{2}\right]=[m(X)+m(Y)$

$-k, m(X)+m(Y)+k]$

$$
\text { where } k=\left\{\frac{\left(y_{2}+x_{2}\right)-\left(y_{1}+x_{1}\right)}{2}\right\}
$$

(ii) Subtraction :

$$
X-Y=\left[x_{1}, x_{2}\right]-\left[y_{1}, y_{2}\right]=[m(X)-m(Y)
$$

$-k, m(X)-m(Y)+k]$

$$
\text { where } k=\left\{\frac{\left(y_{2}+x_{2}\right)-\left(y_{1}+x_{1}\right)}{2}\right\}
$$

\section{(iii)Multiplication :}

$X \cdot Y=\left[x_{1}, x_{2}\right] \cdot\left[y_{1}, y_{2}\right]=[m(X) \cdot m(Y)-k$, $m(X) \cdot m(Y)+k]$

where $k=\min \{(m(X) \cdot \mathrm{m}(\mathrm{Y}))-\alpha, \beta-(m(X) \cdot \mathrm{m}(\mathrm{Y}))\}$ $\alpha=\min \left(x_{1} y_{1}, x_{1} y_{2}, x_{2} y_{1}, x_{2} y_{2}\right)$ and

$\beta=\max \left(x_{1} y_{1}, x_{1} y_{2}, x_{2} y_{1}, x_{2} y_{2}\right)$

(iv) Division :

$$
\frac{1}{X}=\frac{1}{\left[x_{1}, x_{2}\right]}=\left[\frac{1}{m(X)}-k, \frac{1}{m(X)}+k\right]
$$

where $k=\min \left\{\frac{1}{x_{2}}\left(\frac{x_{2}-x_{1}}{x_{1}+x_{2}}\right), \frac{1}{x_{1}}\left(\frac{x_{2}-x_{1}}{x_{1}+x_{2}}\right)\right\}$

and $0 \notin\left[x_{1}, x_{2}\right]$

\section{Interval Matrix}

An interval matrix $A^{I}$ is a matrix whose elements are intervals.

$A^{I}=\left(\begin{array}{ll}{[1,2]} & {[3,4]} \\ {[5,6]} & {[4,7]}\end{array}\right): A^{I}=[\underline{A}, \bar{A}]=$

$\{A ; \underline{A} \leq A \leq \bar{A}\}$

Here, $\underline{A}=\left(\begin{array}{ll}1 & 3 \\ 5 & 4\end{array}\right), \bar{A}=\left(\begin{array}{ll}2 & 4 \\ 6 & 7\end{array}\right)$

\subsection{General Interval Matrix}

$A^{I}=\left(\begin{array}{cccc}a_{11}^{I} & a_{12}^{I} & \ldots & a_{1 n}^{I} \\ a_{21}^{I} & a_{22}^{I} & \ldots & a_{2 n}^{I} \\ \ldots & \ldots & \ldots & \ldots \\ a_{m 1}^{I} & a_{m 2}^{I} & \ldots & a_{m n}^{I}\end{array}\right)=\left(a_{i j}^{I}\right)_{1 \leq i \leq m, 1 \leq j \leq n}$

2.2 Mid Point of a General Interval Matrix

$m\left(A^{I}\right)=\left(\begin{array}{cccc}m\left(a_{11}^{I}\right) & m\left(a_{12}^{I}\right) & \ldots & m\left(a_{1 n}^{I}\right) \\ m\left(a_{21}^{I}\right) & m\left(a_{22}^{I}\right) & \ldots & m\left(a_{2 n}^{I}\right) \\ \ldots & \ldots & \ldots & \ldots \\ m\left(a_{m 1}^{I}\right) & m\left(a_{m 2}^{I}\right) & \ldots & m\left(a_{m n}^{I}\right)\end{array}\right)$

\subsection{Some Interval Matrix Operations}

If $A^{I}, B^{I} \in \mathbb{D}^{m \times n}, X^{I} \in \mathbb{D}^{n}, \alpha^{I} \in \mathbb{D}$

- $\alpha^{I} A^{I}=\alpha^{I}\left(a_{i j}^{I}\right)_{1 \leq i \leq m, 1 \leq j \leq n}$

- $A^{I}+B^{I}=\left(a_{i j}^{I}+b_{i j}^{I}\right)_{1 \leq i \leq m, 1 \leq j \leq n}$

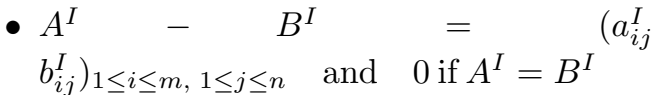

- $A^{I} B^{I}=\left(\sum_{k=1}^{n} a_{i k}^{I} b_{k j}^{I}\right)_{1 \leq i \leq m, 1 \leq j \leq n}$

- $A^{I} X^{I}=\left(\sum_{j=1}^{n} a_{i j}^{I} X^{I}\right)_{1 \leq i \leq m}$

\section{Properties of an Interval Ma- trix}

The determinant of a square interval matrix is an interval number.

i.e. $\operatorname{det} A^{I}=\left|A^{I}\right|=\sum a_{i j}^{I} A_{i j}^{I}$, where $A_{i j}^{I}$ is the cofactor of $a_{i j}^{I}$ with usual meaning.

Definition 3.1. A square interval matrix $A^{I}$ is said to be non singular or regular if $\left|A^{I}\right|$ is invertible (i.e. $\left.0 \notin\left|A^{I}\right|\right)$. Alternatively, a square interval matrix $A^{I}$ is said to be invertible if $\left|A^{I}\right|$ is invertible (i.e. $\left.0 \notin\left|A^{I}\right|\right)$.

Definition 3.2. Let $A^{I}$ be a square interval matrix. The adjoint matrix $A^{I *}$ of $A^{I}$ is the transpose of the matrix of cofactors of the elements of $A^{I}$. That is $A^{I *}=\operatorname{adj}\left(A^{I}\right)=\left(b_{i j}^{I}\right)$, where $b_{i j}^{I}=\left|A_{j i}^{I}\right|$, for all $i, j=1,2,3, \ldots ., n$.

Definition 3.3. For any $A^{I} \in \mathbb{I}^{n \times n}$ if $\left|A^{I}\right|$ is invertible, then the common solution of equations $A^{I} X^{I}$ $=I^{I}$ and $X^{I} A^{I}=I^{I}$ is called the inverse of $A^{I}$ and is denoted by

$A^{I^{-1}}=\frac{\operatorname{adj}\left(A^{I}\right)}{\left|A^{I}\right|}$

If $A^{I}$ is invertible, then $m\left(A^{I^{-1}}\right)=\left[m\left(A^{I}\right)\right]^{-1}$.

Theorem 3.1. let $A^{I *}$ be the adjoint matrix of $A^{I}$ then $A^{I} A^{I *}=A^{I *} A^{I}=\left|A^{I}\right| I^{I}$.

Proof. let $A^{I}=\left(a_{i j}\right)^{I}, A^{I *}=\left(b_{i j}\right)^{I}$ so that $\left(b_{i j}\right)^{I}=$ $\left(A_{j i}\right)^{I}$. Then for $i, j=1,2,3 \ldots, n$, we have

$\left(A^{I} A^{I *}\right)_{i j}=\sum_{k=1}^{n} a_{i k}^{I} b_{k j}^{I}=\sum_{k=1}^{n} a_{i k}^{I} A_{j k}^{I}=\left|A^{I}\right| \delta_{i j}^{I}=\left|A^{I}\right| I^{I}$

and

$\left(A^{I *} A^{I}\right)_{i j}=\sum_{k=1}^{n} b_{i k}^{I} a_{k j}^{I}=\sum_{k=1}^{n} a_{k j}^{I} A_{k i}^{I}=\left|A^{I}\right| \delta_{j i}^{I}=\left|A^{I}\right| I^{I}$

$\delta_{j i}^{I}=\left\{\begin{array}{ll}1 & i=j \\ 0 & i \neq j\end{array}\right\}$

from both the equations we see that $A^{I} A^{I *}=$ $A^{I *} A^{I}=\left|A^{I}\right| I^{I}$ 
Theorem 3.2. (i) If $A^{I}$ is invertible, then the matrix equation $A^{I} X^{I}=I^{I}$ and $A^{I} X^{I}=I^{I}$ both possesses a common solution $X^{I}=\operatorname{adj}\left(A^{I}\right) /$ $\left|A^{I}\right|$.

(ii) If atleast one of the equation is solvable for $X^{I}$ then $\left|A^{I}\right|$ is invertible and so both equations are solvable and possesses a common solution $X^{I}=$ $\operatorname{adj}\left(A^{I}\right) /\left|A^{I}\right|$.

Proposition 3.1. let $A^{I}=[\underline{A}, \bar{A}] \in \mathbb{R}^{n \times n}$ if $\underline{A}$ and $\bar{A}$ are regular and $\underline{A}^{-1} \geq 0, \bar{A}^{-1} \geq 0$ then $A^{I}$ is regular and $A^{I^{-1}}=\left[\underline{A}^{-1}, \bar{A}^{-1}\right] \geq 0$.

\section{Example: Inverse of an Inter- val Matrix}

Calculate inverse of the given interval matrix.

$A^{I}=\left(\begin{array}{ll}{[1,3]} & {[0,1]} \\ {[0,1]} & {[3,5]}\end{array}\right)$

First we check that this interval matrix is regular or not. So we find determinant of the given interval matrix by using modified interval arithemetic.

$\left|A^{I}\right|=[1,3][3,5]-[0,1][0,1]$

let $a_{1}^{I}=[1,3], a_{2}^{I}=[3,5], a_{3}^{I}=[0,1]$,

$a_{4}^{I}=[0,1]$

$\mathrm{m}\left(a_{1}^{I}\right)=2, \mathrm{~m}\left(a_{2}^{I}\right)=4, \mathrm{~m}\left(a_{3}^{I}\right)=1 / 2, \mathrm{~m}\left(a_{4}^{I}\right)=1 / 2$

$a_{1}^{I} \quad a_{2}^{I}=[1,3][3,5], \alpha=\min \{3,5,9,15\}=3$,

$\beta=\max \{3,5,9,15\}=15$

$\mathrm{k}=\min \{8-3,15-8\}=5$

$\left[m\left(a_{1}^{I}\right) \cdot m\left(a_{2}^{I}\right)-k, m\left(a_{1}^{I}\right) \cdot m\left(a_{2}^{I}\right)+k\right]=[3,13]$

same as $[0,1][0,1]=[0,1 / 2]$

$[3,13]-[0,1 / 2]=[5 / 2,13]>0$

so this is a regular interval matrix.

$A^{I^{-1}}=\frac{\operatorname{adj}\left(A^{I}\right)}{\left|A^{I}\right|}$

$A^{I^{-1}}=\frac{1}{[5 / 2,13]} \times\left(\begin{array}{cc}{[3,5]} & {[-1,0]} \\ {[-1,0]} & {[1,3]}\end{array}\right)$

by using modified interval arithemetic-

$\frac{1}{[5 / 2,13]}=\left[\frac{31}{(13 \times 31)}, \frac{73}{(13 \times 31)}\right]$

$A^{I-1}=\left(\begin{array}{cc}{[3,5] \times\left[\frac{31}{(13 \times 31)}, \frac{73}{(13 \times 31)}\right]} & {[-1,0] \times\left[\frac{31}{(13 \times 31)}, \frac{73}{(13 \times 31)}\right]} \\ {[-1,0] \times\left[\frac{31}{(13 \times 31)}, \frac{73}{(13 \times 31)}\right]} & {[1,3] \times\left[\frac{31}{(13 \times 31)}, \frac{73}{(13 \times 31)}\right]}\end{array}\right)$

$[3,5] \times\left[\frac{31}{(13 \times 31)}, \frac{73}{(13 \times 31)}\right]=\left[\frac{93}{(13 \times 31)}, \frac{323}{(13 \times 31)}\right]$

$[-1,0] \times\left[\frac{31}{(13 \times 31)}, \frac{73}{(13 \times 31)}\right]=\left[\frac{-52}{(13 \times 31)}, 0\right]$

$[1,3] \times\left[\frac{31}{(13 \times 31)}, \frac{73}{(13 \times 31)}\right]=\left[\frac{31}{(13 \times 31)}, \frac{177}{(13 \times 31)}\right]$

$\mathrm{A}^{I-1}=\left(\begin{array}{c}{\left[\frac{93}{(13 \times 31)}, \frac{323}{(13 \times 31)}\right]} \\ {\left[\frac{-52}{(13 \times 31)}, 0\right]}\end{array}\right.$

$\left.\begin{array}{c}{\left[\frac{-52}{(13 \times 31)}, 0\right]} \\ {\left[\frac{31}{(13 \times 31)}, \frac{177}{(13 \times 31)}\right]}\end{array}\right)$

\section{MATLAB Code For Inverse Of Interval Matrix}

function $\mathrm{Z}=$ invs1 $(\mathrm{a}, \mathrm{b}, \mathrm{c}, \mathrm{d}, \mathrm{e}, \mathrm{f}, \mathrm{g}, \mathrm{h})$

$\mathrm{i}=(\mathrm{a}+\mathrm{b}) / 2$;

$\mathrm{j}=(\mathrm{g}+\mathrm{h}) / 2$;

$\mathrm{k}=[\mathrm{a} * \mathrm{~g}, \mathrm{a} * \mathrm{~h}, \mathrm{~b} * \mathrm{~g}, \mathrm{~b} * \mathrm{~h}]$;

$\mathrm{l}=\min (\mathrm{k})$;

$\mathrm{m}=\max (\mathrm{k})$;

$\mathrm{n}=[\mathrm{i} * \mathrm{j}-\mathrm{l}, \mathrm{m}-\mathrm{i} * \mathrm{j}]$;

$\mathrm{o}=\min (\mathrm{n})$;

$\mathrm{p}=[\mathrm{i} * \mathrm{j}-\mathrm{o}, \mathrm{i} * \mathrm{j}+\mathrm{o}]$;

$\mathrm{q}=(\mathrm{c}+\mathrm{d}) / 2$;

$\mathrm{r}=(\mathrm{e}+\mathrm{f}) / 2$;

$\mathrm{s}=[\mathrm{c} * \mathrm{e}, \mathrm{c} * \mathrm{f}, \mathrm{d} * \mathrm{e}, \mathrm{d} * \mathrm{f}]$;

$\mathrm{t}=\min (\mathrm{s})$;

$\mathrm{u}=\max (\mathrm{s})$;

$\mathrm{v}=[\mathrm{q} * \mathrm{r}-\mathrm{t}, \mathrm{u}-\mathrm{q} * \mathrm{r}]$;

$\mathrm{w}=\min (\mathrm{v})$;

$\mathrm{x}=[\mathrm{q} * \mathrm{r}-\mathrm{w}, \mathrm{q} * \mathrm{r}+\mathrm{w}]$

$\mathrm{z}=((\mathrm{i} * \mathrm{j}-\mathrm{o})+(\mathrm{i} * \mathrm{j}+\mathrm{o})) / 2$;

$\mathrm{A}=((\mathrm{q} * \mathrm{r}-\mathrm{w})+(\mathrm{q} * \mathrm{r}+\mathrm{w})) / 2$;

$\mathrm{B}=$

$(((\mathrm{i} * \mathrm{j}+\mathrm{o})+(\mathrm{q} * \mathrm{r}+\mathrm{w}))-((\mathrm{i} * \mathrm{j}-\mathrm{o})+(\mathrm{q} * \mathrm{r}-\mathrm{w}))) / 2$;

$\mathrm{C}=[\mathrm{z}-\mathrm{A}-\mathrm{B}, \mathrm{z}-\mathrm{A}+\mathrm{B}]$;

$\mathrm{D}=[\mathrm{g}, \mathrm{h},-\mathrm{d},-\mathrm{c},-\mathrm{f},-\mathrm{e}, \mathrm{a}, \mathrm{b}]$;

$\mathrm{E}=(\mathrm{z}-\mathrm{A}-\mathrm{B}+\mathrm{z}-\mathrm{A}+\mathrm{B}) / 2$;

$\mathrm{a} 1=(1 /(\mathrm{z}-\mathrm{A}+\mathrm{B})) *((\mathrm{z}-\mathrm{A}+\mathrm{B})-(\mathrm{z}-\mathrm{A}-\mathrm{B}))$

$/((\mathrm{z}-\mathrm{A}+\mathrm{B})+(\mathrm{z}-\mathrm{A}-\mathrm{B}))$;

$\mathrm{a} 2=(1 /(\mathrm{z}-\mathrm{A}-\mathrm{B})) *((\mathrm{z}-\mathrm{A}+\mathrm{B})-(\mathrm{z}-\mathrm{A}-\mathrm{B}))$

$/((\mathrm{z}-\mathrm{A}+\mathrm{B})+(\mathrm{z}-\mathrm{A}-\mathrm{B}))$;

$\mathrm{F}=[\mathrm{a} 1, \mathrm{a} 2]$

$\mathrm{G}=\min (\mathrm{F})$;

$\mathrm{H}=[(1 / \mathrm{E})-\mathrm{G},(1 / \mathrm{E})+\mathrm{G}]$;

$\mathrm{I}=(((1 / \mathrm{E})-\mathrm{G})+((1 / \mathrm{E})+\mathrm{G})) / 2$;

$\mathrm{J}=(\mathrm{g}+\mathrm{h}) / 2$;

$\mathrm{K}=[((1 / \mathrm{E})-\mathrm{G}) * \mathrm{~g},((1 / \mathrm{E})-\mathrm{G}) * \mathrm{~h}$,

$((1 / \mathrm{E})+\mathrm{G}) * \mathrm{~g},((1 / \mathrm{E})+\mathrm{G}) * \mathrm{~h}]$;

$\mathrm{L}=\min (\mathrm{K})$;

$\mathrm{M}=\max (\mathrm{K})$;

$\mathrm{N}=[\mathrm{I} * \mathrm{~J}-\mathrm{L}, \mathrm{M}-\mathrm{I} * \mathrm{~J}]$;

$\mathrm{O}=\min (\mathrm{N})$;

$\mathrm{P}=[\mathrm{I} * \mathrm{~J}-\mathrm{O}, \mathrm{I} * \mathrm{~J}+\mathrm{O}]$;

$\mathrm{Q}=(-\mathrm{d}-\mathrm{c}) / 2$;

$\mathrm{R}=[((1 / \mathrm{E})-\mathrm{G}) *(-\mathrm{d}),((1 / \mathrm{E})-\mathrm{G}) *(-\mathrm{c})$,

$((1 / \mathrm{E})+\mathrm{G}) *(-\mathrm{d}),((1 / \mathrm{E})+\mathrm{G}) *(-\mathrm{c})]$;

$\mathrm{S}=\min (\mathrm{R})$;

$\mathrm{T}=\max (\mathrm{R})$;

$\mathrm{U}=[\mathrm{I} * \mathrm{Q}-\mathrm{S}, \mathrm{T}-\mathrm{I} * \mathrm{Q}]$;

$\mathrm{V}=\min (\mathrm{U})$;

$\mathrm{W}=[\mathrm{I} * \mathrm{Q}-\mathrm{V}, \mathrm{I} * \mathrm{Q}+\mathrm{V}]$;

$\mathrm{X}=(-\mathrm{f}-\mathrm{e}) / 2$;

$\mathrm{Y}=[((1 / \mathrm{E})-\mathrm{G}) *(-\mathrm{f}),((1 / \mathrm{E})-\mathrm{G}) *(-\mathrm{e})$, $((1 / \mathrm{E})+\mathrm{G}) *(-\mathrm{f}),((1 / \mathrm{E})+\mathrm{G}) *(-\mathrm{e})]$;

aa $=\min (\mathrm{Y})$;

$\mathrm{ab}=\max (\mathrm{Y})$

$\mathrm{ac}=[\mathrm{I} * \mathrm{X}-\mathrm{aa}, \mathrm{ab}-\mathrm{I} * \mathrm{X}]$; 
$\mathrm{ad}=\min (\mathrm{ac})$;

ae $=[\mathrm{I} * \mathrm{X}-\mathrm{ad}, \mathrm{I} * \mathrm{X}+\mathrm{ad}] ;$

af $=(\mathrm{a}+\mathrm{b}) / 2$;

$\mathrm{ag}=[((1 / \mathrm{E})-\mathrm{G}) * \mathrm{a},((1 / \mathrm{E})-\mathrm{G}) * \mathrm{~b}$,

$((1 / \mathrm{E})+\mathrm{G}) * \mathrm{a},((1 / \mathrm{E})+\mathrm{G}) * \mathrm{~h}]$;

$\mathrm{ah}=\min (\mathrm{ag})$;

$\mathrm{ai}=\max (\mathrm{ag})$;

$\mathrm{aj}=[\mathrm{I} * \mathrm{af}-\mathrm{ah}, \mathrm{ai}-\mathrm{I} * \mathrm{af}] ;$

$\mathrm{ak}=\min (\mathrm{aj})$;

al $=[\mathrm{I} * \mathrm{af}-\mathrm{ak}, \mathrm{I} * \mathrm{af}+\mathrm{ak}]$;

$\mathrm{Z}=[\mathrm{P}, \mathrm{W} ; \mathrm{ae}, \mathrm{al}]$;

end

$>\mathrm{Z}=\operatorname{invs} 1(1,3,0,1,0,1,3,5)$

$\mathrm{Z}=$

$$
\begin{aligned}
& 0.2308 \quad 0.8015 \\
& -0.1290 \\
& -0.1290 \\
& 0 \\
& 0 \quad 0.0769
\end{aligned}
$$

0.4392

$>\mathrm{A}=$ evalin (symengine, 'Dom: :

Interval ([0.2308, 0.8015$]) ')$

$\mathrm{A}=$

$[0.2308,0.8015]$

$>\mathrm{B}=$ evalin (symengine, 'Dom: :

Interval $\left.([-0.1290,0])^{\prime}\right)$

$\mathrm{B}=$

$[-0.129,0]$

$>>\mathrm{C}=$ evalin (symengine, 'Dom: :

Interval $\left.([-0.1290,0])^{\prime}\right)$

$\mathrm{C}=$

$[-0.129,0]$

$>\mathrm{D}=$ evalin ( symengine, 'Dom: :

Interval ([0.0769, 0.4392$]) ')$

$\mathrm{D}=$

$[0.0769,0.4392]$

$>\mathrm{E}=[\mathrm{A}, \mathrm{B} ; \mathrm{C}, \mathrm{D}]$

$\mathrm{E}=$

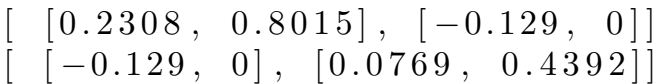

let us consider an example :

$A^{I}=\left(\begin{array}{ccc}{[3.7,4.3]} & {[-1.5,-0.5]} & {[0,0]} \\ {[-1.5,-0.5]} & {[3.7,4.3]} & {[-1.5,-0.5]} \\ {[0,0]} & {[-1.5,-0.5]} & {[3.7,4.3]}\end{array}\right)$,
$b^{I}=\left(\begin{array}{c}{[-14,14]} \\ {[-9,9]} \\ {[-3,3]}\end{array}\right)$

$\left|A^{I}\right|=[37.103,74.897]$

$\left|A^{I(1)}\right|=\left|\begin{array}{ccc}{[-14,14]} & {[-1.5,-0.5]} & {[0,0]} \\ {[-9,9]} & {[3.7,4.3]} & {[-1.5,-0.5]} \\ {[-3,3]} & {[-1.5,-0.5]} & {[3.7,4.3]}\end{array}\right|=$

$\left|A^{I(2)}\right|=\left|\begin{array}{ccc}{[3.7,4.3]} & {[-14,14]} & {[0,0]} \\ {[-1.5,-0.5]} & {[-9,9]} & {[-1.5,-0.5]} \\ {[0,0]} & {[-3,3]} & {[3.7,4.3]}\end{array}\right|=$

$[-271.86,271.86]$

$\left|A^{I(3)}\right|=\left|\begin{array}{ccc}{[3.7,4.3]} & {[-1.5,-0.5]} & {[-14,14]} \\ {[-1.5,-0.5]} & {[3.7,4.3]} & {[-9,9]} \\ {[0,0]} & {[-1.5,-0.5]} & {[-3,3]}\end{array}\right|=$

$[-144.77,144.77]$

then by the theorem -

$$
\begin{aligned}
& x_{1}^{I}=\frac{\left|A^{I(1)}\right|}{\left|A^{I}\right|}=[-6.988,6.988] \\
& x_{2}^{I}=\frac{\left|A^{I(2)}\right|}{\left|A^{I}\right|}=[-5.981,5.981] \\
& x_{3}^{I}=\frac{\left|A^{I(3)}\right|}{\left|A^{I}\right|}=[-3.185,3.185]
\end{aligned}
$$




\subsection{Jacobi Iterative Method}

let $A^{I} X^{I}=B^{I}$; where $A^{I}$ is an interval matrix and $X^{I}$ and $B^{I}$ are interval vectors.

$\begin{aligned} A^{I} & =\left(\begin{array}{cccc}a_{11}^{I} & a_{12}^{I} & \ldots & a_{1 n}^{I} \\ a_{21}^{I} & a_{22}^{I} & \ldots & a_{2 n}^{I} \\ \ldots & \ldots & \ldots & \ldots \\ a_{n 1}^{I} & a_{n 2}^{I} & \ldots & a_{n n}^{I}\end{array}\right)=\left(a_{i j}^{I}\right)_{1 \leq i \leq n, 1 \leq j \leq n} \\ X^{I} & =\left(\begin{array}{c}x_{1}^{I} \\ x_{2}^{I} \\ \ldots \\ x_{n}^{I}\end{array}\right)=\left(x_{i}^{I}\right)_{1 \leq i \leq n} \\ B^{I} & =\left(\begin{array}{c}b_{1}^{I} \\ b_{2}^{I} \\ \ldots \\ b_{n}^{I}\end{array}\right)=\left(b_{i}^{I}\right)_{1 \leq i \leq n}\end{aligned}$

Iterations :

$x_{1}^{I(n+1)}=\frac{b_{1}^{I}}{a_{11}^{I}}-\frac{a_{12}^{I} x_{2}^{I(n)}}{a_{11}^{I}}-\ldots-\frac{a_{1 n}^{I} x_{n}^{I(n)}}{a_{11}^{I}}$

$x_{2}^{I(n+1)}=\frac{b_{2}^{I}}{a_{22}^{I}}-\frac{a_{21}^{I} x_{1}^{I(n)}}{a_{22}^{I}}-\ldots-\frac{a_{2 n}^{I} x_{n}^{I(n)}}{a_{22}^{I}}$

$x_{n}^{I(n+1)}=\frac{b_{n}^{I}}{a_{n n}^{I}}-\frac{a_{n 1}^{I} x_{1}^{I(n)}}{a_{n n}^{I}}-\ldots-\frac{a_{n, n-1}^{I} x_{n-1}^{I(n)}}{a_{n n}^{I}}$

\section{Example :}

$[3.7,4.3] x_{1}^{I}+[-1.5,-0.5] x_{2}^{I}+[0,0] x_{3}^{I}=[-14,14]$

$[-1.5,-0.5] x_{1}^{I}+[3.7,4.3] x_{2}^{I}+[-1.5,-0.5] x_{3}^{I}=[-9,9]$

$[0,0] x_{1}^{I}+[-1.5,-0.5] x_{2}^{I}+[3.7,4.3] x_{3}^{I}=[-3,3]$

First Iteration :

first we take $x_{1}^{I(0)}=[0,0], x_{2}^{I(0)}=[0,0], x_{3}^{I(0)}=[0,0]$

$x_{1}^{I(1)}=\frac{1}{[3.7,4.3]} \times\left([-14,14]-[-1.5,-0.5] x_{2}^{I(0)}\right.$

$\left.-[0,0] x_{3}^{I(0)}\right)$

$x_{1}^{I(1)}=\frac{1}{[3.7,4.3]} \times([-14,14])=[-3.7436,3.7436]$

(by using modified interval arithemetic)

$x_{2}^{I(1)}=\frac{1}{[3.7,4.3]} \times\left([-9,9]-[-1.5,-0.5] x_{1}^{I(0)}\right.$

$\left.-[-1.5,-0.5] x_{3}^{I(0)}\right)$

$x_{2}^{I(1)}=\frac{1}{[3.7,4.3]} \times([-9,9])=[-2.4066,2.4066]$

$x_{3}^{I(1)}=\frac{1}{[3.7,4.3]} \times\left([-3,3]-[-1.5,-0.5] x_{2}^{I(0)}\right.$

$\left.-[0,0] x_{3}^{I(0)}\right)$

$x_{3}^{I(1)}=\frac{1}{[3.7,4.3]} \times([-3,3])=[-0.8022,0.8022]$

Second Iteration :

$x_{1}^{I(2)}=\frac{1}{[3.7,4.3]} \times\left([-14,14]-[-1.5,-0.5] x_{2}^{I(1)}\right.$

$\left.-[0,0] x_{3}^{I(1)}\right)$

$x_{1}^{I(2)}=[0.2326,0.2674] \times([-14,14]-[-1.5,-0.5]$

$\times[-2.4066,2.4066]-[0,0] \times[-0.8022,0.8022])$

$x_{1}^{I(2)}=[-4.7089,4.7089]$

$x_{2}^{I(2)}=\frac{1}{[3.7,4.3]} \times\left([-9,9]-[-1.5,-0.5] x_{1}^{I(1)}\right.$

$\left.-[-1.5,-0.5] x_{3}^{I(1)}\right)$

$x_{2}^{I(2)}=[0.2326,0.2674] \times([-9,9]-[-1.5,-0.5]$

$\times[-3.7436,3.7436]-[-1.5,-0.5] \times[-0.8022,0.8022])$

$x_{1}^{I(2)}=[-4.2299,4.2299]$
$x_{3}^{I(2)}=\frac{1}{[3.7,4.3]} \times\left([-3,3]-[-1.5,-0.5] \mathrm{x}_{2}^{I(1)}-[0,0] x_{1}^{I(1)}\right)$

$x_{3}^{I(2)}=[0.2326,0.2674] \times([-3,3]-[-1.5,-0.5]$

$\times[-2.4066,2.4066]-[0,0] \times[-3.7436,3.7436])$

$x_{3}^{I(2)}=[-1.7675,1.7675]$

Third Iteration :

$x_{1}^{I(3)}=\frac{1}{[3.7,4.3]} \times\left([-14,14]-[-1.5,-0.5] x_{2}^{I(2)}-[0,0] x_{3}^{I(2)}\right)$

$x_{1}^{I(2)}=[0.2326,0.2674] \times([-14,14]-[-1.5,-0.5]$

$\times[-4.2299,4.2299]-[0,0] \times[-1.7675,1.7675])$

$x_{1}^{I(3)}=[-5.4402,5.4402]$

$x_{2}^{I(3)}=\frac{1}{[3.7,4.3]} \times\left([-9,9]-[-1.5,-0.5] x_{1}^{I(2)}-\right.$

$\left.[-1.5,-0.5] x_{3}^{I(2)}\right)$

$x_{2}^{I(3)}=[0.2326,0.2674] \times([-9,9]-[-1.5,-0.5] \times$

$[-4.7089,4.7089]-[-1.5,-0.5] \times[-1.7675,1.7675])$

$x_{2}^{I(3)}=[-5.0043,5.0043]$

$x_{3}^{I(3)}=\frac{1}{[3.7,4.3]} \times\left([-3,3]-[-1.5,-0.5] x_{2}^{I(2)}-[0,0] x_{1}^{I(2)}\right)$

$x_{3}^{I(3)}=[0.2326,0.2674] \times([-3,3]-[-1.5,-0.5] \times$

$[-4.2299,4.2299]-[0,0] \times[-4.7089,4.7089])$

$x_{3}^{I(3)}=[-2.4961,2.4986]$

after three iterations we get the solution -

$x_{1}^{I}=[-5.4402,5.4402]$

$x_{2}^{I}=[-5.0043,5.0043]$

$x_{3}^{I}=[-2.4961,2.4986]$

\section{Convergence of Jacobi Method :}

for convergence, matrix will be digonally dominant.

$\left|a_{11}^{I}\right| \geq\left|a_{12}^{I}\right|+\left|a_{13}^{I}\right|$

$\left|a_{22}^{I}\right| \geq\left|a_{21}^{I}\right|+\left|a_{23}^{I}\right|$

$\left|a_{33}^{I}\right| \geq\left|a_{31}^{I}\right|+\left|a_{32}^{I}\right|$

$\min \left|a_{11}^{I}\right|=3.7 ; \max \left|a_{12}^{I}\right|+\max \left|a_{13}^{I}\right|=1.5+0=1.5 ;$

$\min \left|a_{11}^{I}\right|>\max \left|a_{12}^{I}\right|+\max \left|a_{13}^{I}\right|$ this implies $\left|a_{11}^{I}\right|>\left|a_{12}^{I}\right|+\left|a_{13}^{I}\right|$ in all cases.

simillarly,

$\min \left|a_{22}^{I}\right|=3.7 ; \max \left|a_{21}^{I}\right|+\max \left|a_{23}^{I}\right|=1.5+1.5=3 ;$

$\min \left|a_{22}^{I}\right|>\max \left|a_{21}^{I}\right|+\max \left|a_{23}^{I}\right|$

$\min \left|a_{33}^{I}\right|=3.7 ; \max \left|a_{31}^{I}\right|+\max \left|a_{32}^{I}\right|=0+1.5=1.5 ;$

$\min \left|a_{33}^{I}\right|>\max \left|a_{31}^{I}\right|+\max \left|a_{32}^{I}\right|$

so matrix is digonally dominant. so this is convergent

\subsection{Crout Method}

$A^{I} X^{I}=B^{I}$

$M^{I^{\prime}}=\left[A^{I^{\prime}}: B^{\left.I^{\prime}\right]}\right.$; here $M^{I^{\prime}}$ is a Auxiliary Matrix.

$M^{I^{\prime}}=\left(\begin{array}{cccccc}a_{11}^{I}{ }^{\prime} & a_{12}^{I}{ }^{\prime} & \ldots & a_{1 n}^{I}{ }^{\prime}, & : & b_{1}^{I^{\prime}} \\ a_{21}^{I}{ }^{\prime} & a_{22}^{I}{ }^{\prime} & \ldots & a_{2 n}^{I}{ }^{\prime} & : & b_{2}^{I^{\prime}} \\ \ldots, & \ldots, & \ldots & \ldots, & : & : \\ a_{n 1}^{I}{ }^{\prime} & a_{n 2}^{I}{ }^{\prime} & \ldots & a_{n n}^{I}{ }^{\prime} & : & b_{n}^{I^{\prime}}\end{array}\right)=$

$\left(a_{i j}^{I}{ }^{\prime}\right) 1 \leq i \leq n, 1 \leq j \leq n$

Methodology :

- when $\mathrm{i} \geq j ; a_{i j}{ }^{{ }^{\prime}}=a_{i j}^{I}-\sum_{k=1}^{j-1} a_{i k}^{I}{ }^{\prime} a_{k j}^{I}{ }^{\prime}$ when $\mathrm{i}<\mathrm{j} ; a_{i j}^{I}{ }^{\prime}=\left(a_{i j}^{I}-\sum_{k=1}^{i-1} a_{i k}^{I}{ }^{\prime} a_{k j}^{I}{ }^{\prime}\right) \times \frac{1}{a_{i i}^{I}{ }^{\prime}}$

- $b_{i}^{I^{\prime}}=\left(b_{i}^{I}-\sum_{k=1}^{i-1} a_{i k}^{I}{ }^{\prime} b_{k}^{I^{\prime}}\right) \times \frac{1}{a_{i i}^{I}{ }^{\prime}}$

- $x_{i}^{I}=b_{i}^{I^{\prime}}-\sum_{k=i+1}^{n} a_{i k}^{I}{ }^{\prime} x_{k}^{I}$

Example :

$A^{I}=\left(\begin{array}{ccc}{[3.7,4.3]} & {[-1.5,-0.5]} & {[0,0]} \\ {[-1.5,-0.5]} & {[3.7,4.3]} & {[-1.5,-0.5]} \\ {[0,0]} & {[-1.5,-0.5]} & {[3.7,4.3]}\end{array}\right), \quad b^{I}=$ 
$\left(\begin{array}{c}{[-14,14]} \\ {[-9,9]} \\ {[-3,3]}\end{array}\right)$

apply above process - we get

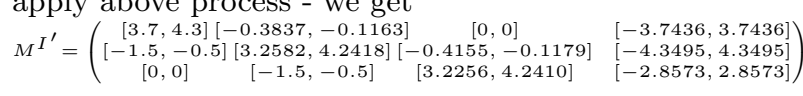

$x_{3}^{I}=b_{3}^{I^{\prime}}=[-2.8513,2.8573]$

$x_{2}^{I}=b_{2}^{I^{\prime}}-a_{23}^{I^{\prime}} x_{3}^{I}=[-5.5367,5.5367]$

$x_{1}^{I}=b_{1}^{I^{\prime}}-a_{12}^{I}{ }^{\prime} \quad x_{2}^{I}-a_{13}^{I}{ }^{\prime} x_{3}^{I}=[-5.8680,5.8680]$

\subsection{Cholesky Method}

let $A^{I} X^{I}=B^{I}$

Interval Matrix $A^{I}$ can be written as product of lower and upper triangular interval matrix.

$$
\begin{aligned}
& \left(\begin{array}{cccc}
a_{11}^{I} & a_{12}^{I} & \ldots & a_{1 n}^{I} \\
a_{21}^{I} & a_{22}^{I} & \ldots & a_{2 n}^{I} \\
\ldots & \ldots & \ldots & \ldots \\
a_{n 1}^{I} & a_{n 2}^{I} & \ldots & a_{n n}^{I}
\end{array}\right)=\left(\begin{array}{cccc}
l_{11}^{I} & 0 & \ldots & 0 \\
l_{21}^{I} & l_{22}^{I} & \ldots & 0 \\
\ldots & \ldots & \ldots & \ldots \\
l_{n 1}^{I} & l_{n 2}^{I} & \ldots & l_{n n}^{I}
\end{array}\right) \\
& \times\left(\begin{array}{cccc}
1 & l_{12}^{I} & \ldots & l_{1 n}^{I} \\
0 & 1 & \ldots & l_{2 n}^{I} \\
\ldots & \ldots & \ldots & \ldots \\
0 & 0 & \ldots & 1
\end{array}\right) \\
& L^{I} U^{I} X^{I}=B^{I} \\
& \operatorname{let} U^{I} X^{I}=C^{I} \Longrightarrow \mathrm{L}^{I} C^{I}=B^{I}
\end{aligned}
$$

Example :

$$
\begin{aligned}
& A^{I} X^{I}=B^{I} \\
& \left(\begin{array}{ccc}
{[3.7,4.3]} & {[-1.5,-0.5]} & {[0,0]} \\
{[-1.5,-0.5]} & {[3.7,4.3]} & {[-1.5,-0.5]} \\
{[0,0]} & {[-1.5,-0.5]} & {[3.7,4.3]}
\end{array}\right) \times\left(\begin{array}{c}
x_{1}^{I} \\
x_{2}^{I} \\
x_{3}^{I}
\end{array}\right) \\
& =\left(\begin{array}{c}
{[-14,14]} \\
{[-9,9]} \\
{[-3,3]}
\end{array}\right) \\
& \left(\begin{array}{ccc}
{[3.7,4.3]} & {[-1.5,-0.5]} & {[0,0]} \\
{[-1.5,-0.5]} & {[3.7,4.3]} & {[-1.5,-0.5]} \\
{[0,0]} & {[-1.5,-0.5]} & {[3.7,4.3]}
\end{array}\right) \\
& =\left(\begin{array}{ccc}
l_{11}^{I} & 0 & 0 \\
l_{21}^{I} & l_{22}^{I} & 0 \\
l_{31}^{I} & l_{32}^{I} & l_{33}^{I}
\end{array}\right) \times\left(\begin{array}{ccc}
1 & l_{12}^{I} & l_{1 n}^{I} \\
0 & 1 & l_{23}^{I} \\
0 & 0 & 1
\end{array}\right) \\
& =\left(\begin{array}{ccc}
l_{11}^{I} & l_{11}^{I} l_{12}^{I} & l_{11}^{I} l_{13}^{I} \\
l_{21}^{I} & l_{21}^{I} l_{12}^{I}+l_{22}^{I} & l_{21}^{I} l_{13}^{I}+l_{22}^{I} l_{23}^{I} \\
l_{31}^{I} & l_{31}^{I} l_{12}^{I}+l_{32}^{I} & l_{31}^{I} l_{13}^{I}+l_{32}^{I} l_{23}^{I}+l_{33}^{I}
\end{array}\right)
\end{aligned}
$$

by comparision

$l_{11}^{I}=[3.7,4.3] ; l_{12}^{I}=[-0.3837,-0.1163] ; l_{13}^{I}=[0,0]$

$l_{21}^{I}=[-1.5,-0.5] ; l_{2 \nmid 2}^{I}[2582,4.2418] ;$

$l_{23}^{I}=[-0.4155,-0.1179]$

$l_{31}^{I}=[0,0] ; l_{32}^{I}=[-1.5,-0.5] ; l_{33}^{I}=[3.2256,4.2256]$

$$
\begin{array}{r}
A^{I}=\left(\begin{array}{ccc}
{[3.7,4.3]} & {[0,0]} & {[0,0]} \\
{[-1.5,-0.5]} & {[3.2582,4.2418]} & {[0,0]} \\
{[0,0]} & {[-1.5,-0.5]} & {[3.2256,4.2410]}
\end{array}\right) \\
\times\left(\begin{array}{ccc}
{[1,1]} & {[-0.3837,-0.1163]} & {[0,0]} \\
{[0,0]} & {[1,1]} & {[-0.4155,-0.1179]} \\
{[0,0]} & {[0,0]} & {[1,1]}
\end{array}\right)
\end{array}
$$

$L^{I} U^{I} X^{I}=\left(\begin{array}{c}{[-14,14]} \\ {[-9,9]} \\ {[-3,3]}\end{array}\right)$

$U^{I} X^{I}=C^{I}$

$L^{I} C^{I}=\left(\begin{array}{c}{[-14,14]} \\ {[-9,9]} \\ {[-3,3]}\end{array}\right)$

$\left(\begin{array}{ccc}{[3.7,4.3]} & {[0,0]} & {[0,0]} \\ {[-1.5,-0.5]} & {[3.2582,4.2418]} & {[0,0]} \\ {[0,0]} & {[-1.5,-0.5]} & {[3.2256,4.2410]}\end{array}\right)$

$\times\left(\begin{array}{c}c_{1}^{I} \\ c_{2}^{I} \\ c_{3}^{I}\end{array}\right)=\left(\begin{array}{c}{[-14,14]} \\ {[-9,9]} \\ {[-3,3]}\end{array}\right)$

solve these equations by using modified interval arithemetic. we get

$\mathrm{c}_{1}^{I}=[-3.7436,3.7436] ; \quad c_{2}^{I}=[-4.3495,4.3495] ;$

$c_{3}^{I}=[-2.8573,2.8573]$

$C^{I}=U^{I} X^{I}$

$\left(\begin{array}{ccc}{[1,1]} & {[-0.3837,-0.1163]} & {[0,0]} \\ {[0,0]} & {[1,1]} & {[-0.4155,-0.1179]} \\ {[0,0]} & {[0,0]} & {[1,1]}\end{array}\right)$

$\times\left(\begin{array}{c}x_{1}^{I} \\ x_{2}^{I} \\ x_{3}^{I}\end{array}\right)$

solving equations we get -

$x_{1}^{I}=[-5.8680,5.8680]$

$x_{2}^{I}=[-5.5367,5.5367]$

$x_{3}^{I}=[-2.8573,2.8573]$

\section{Conclusion}

In this paper, we have derived the inverse of an interval matrix using modified interval arithmetic and developed matlab code. Then, we have calculated solution of system of equations with interval coefficient using Determinant Method, Jacobi Iterative Method, Crout Method and Cholesky Method.

Acknowledgement:I am heartily thankful to Prof. Geetanjali Panda, IIT Kharagpur for her constant encouragement throughout this work. Her valuable advices and guidance were a constant source of inspiration for me.

\section{References}

[1] K Ganesan. "On some properties of interval matrices". In: a a 1 (2007), p. 2.

[2] Ramon E Moore, R Baker Kearfott, and Michael J Cloud. Introduction to interval analysis. Vol. 110. Siam, 2009. 
[3] T Nirmala et al. "Inverse interval matrix: A new approach". In: Applied Mathematical Sciences 5.13 (2011), pp. 607-624.

[4] Jirı Rohn. "Inverse interval matrix". In: SIAM Journal on Numerical Analysis 30.3 (1993), pp. $864-870$. 